\title{
A review on the impact of aircraft cabin air quality and cabin pressure on human wellbeing
}

\begin{abstract}
Airliner cabins present more complicated scenario due to different design and operation challenges owing to the extreme environmental conditions, complexity of the operational systems, and the authorities that govern such environments. The scientific evaluation is rendered difficult due to lack of empirical evidence determining the airliner cabin air quality as well as consequent health effects occurring due to short or long flight exposure. Crew members and passengers report dizziness, fatigue, headaches, sinus and ear problems, dry eyes and sore throats during and after travel. There are persistent concerns about the transmission of infectious agents such as influenza, tuberculosis and measles viruses during flights. Moreover, a systematic collection of data related to airplane environmental exposures is not yet available and the effects of environmental conditions on wellbeing of travelers and their comfort level are yet to be fully ascertained. In this work, a systematic review of the air quality inside the airliner cabin are discussed. The potential pollutants and their established causes are discussed. In addition to this, major health discomforts faced by the occupants are presented.
\end{abstract}

Keyword: Aircraft cabin air quality; Biological agents; Ozone; Volatile organic compounds 